\title{
Effects of inter-resonator coupling in split ring resonator loaded metamaterial transmission lines
}

\author{
J. Naqui, ${ }^{1}$ A. Fernández-Prieto, ${ }^{2}$ F. Mesa, ${ }^{2}$ F. Medina,${ }^{2}$ and F. Martín ${ }^{1}$ \\ ${ }^{1}$ GEMMA/CIMITEC, Departament d'Enginyeria Electrònica, Universitat Autònoma de Barcelona, \\ 08193 Bellaterra, Barcelona, Spain \\ ${ }^{2}$ Grupo de Microondas, Universidad de Sevilla, 41012 Sevilla, Spain
}

(Received 9 January 2014; accepted 17 March 2014; published online 20 May 2014)

\begin{abstract}
This paper investigates the effects of inter-resonator coupling in metamaterial transmission lines loaded with split ring resonators (SRRs). The study is performed from Bloch mode theory applied to the multiport equivalent circuit model of the unit cell of such artificial lines. From this analysis, it follows that the stopband bandwidth, inherent to SRR-loaded lines, is enhanced as inter-resonator coupling strengthens, and this enhancement is attributed to the presence of complex modes. The theoretical results are corroborated through calculation of the dispersion relation using a full-wave eigenmode solver, and also by measuring the frequency response of SRR-loaded lines with different inter-resonator distance and, hence, coupling. (c) 2014 AIP Publishing LLC. [http://dx.doi.org/10.1063/1.4876444]
\end{abstract}

\section{INTRODUCTION}

Transmission lines periodically loaded with split ring resonators (SRRs) ${ }^{1}$ inhibit wave propagation in the vicinity of the SRR fundamental resonance. ${ }^{2,3}$ As long as the SRRs and their spacing are electrically small, these SRR-loaded lines can be considered to be one-dimensional effective media exhibiting a negative effective permeability in a narrow band above SRR resonance (the effective permeability of these structures is described by the Lorenz model ${ }^{4,5}$ ). Actually, the stopband of these lines includes not only the region where the effective permeability is negative but also a narrow band below the resonance frequency of the SRRs where the effective permeability may be interpreted to be highly positive. Additionally, according to Ref. 6, in the event that SRRs are coupled to each other, the effective permeability becomes complex (under lossless conditions) within a region that emerges in the transition from positive to negative values of its real part. Nevertheless, in the present work the interpretation of the stopband is based on the analysis of Bloch mode theory, rather than on the effective permeability.

SRR-loaded lines have been applied to the implementation of stopband filters, where bandwidth has been controlled by slightly varying the dimensions of the SRR array. ${ }^{7}$ The resulting structures can be viewed as quasi-periodic transmission lines where the effective permeability varies along the line. With this strategy, it is clear that the resonance frequency of the different SRRs can be slightly tuned along the desired frequency range, with the result of a broadened stopband. Alternatively, bandwidth can be enhanced by using tightly coupled SRRs. This approach was recently considered in transmission lines periodically loaded with complementary split ring resonators (CSRRs), ${ }^{8}$ formerly proposed in Ref. 9. Subsequently, this approach was applied in Ref. 10 to widen the common mode suppressed band of differential microstrip lines.

In order to achieve significant inter-resonator coupling, the CSRRs in Ref. 8 were chosen to be rectangular-shaped (the long side being oriented along the transversal direction of propagation) and separated by very small distances. As found therein, the relevant feature is that rejection bandwidth enhancement in CSRR-loaded lines with tightly coupled resonators can be related to the presence of complex modes supported by the corresponding periodic infinite structure. These modes, in spite of the absence of losses, have complex propagation constants and appear as conjugate pairs in reciprocal structures. Since the power contribution of the individual modes is in opposite direction to each other, complex modes do not generally carry net power. ${ }^{11-17}$ The existence of these modes in CSRR-loaded lines was demonstrated through Bloch mode theory, by analyzing the equivalent four-port circuit model of the unit cell, and by obtaining the modal solutions through a full-wave eigenmode solver. ${ }^{8}$ Analogously, the theoretical analysis of SRR-loaded lines with coupled resonators carried out in Ref. 6 also leads to a pair of complex propagation constants in the region where a complex effective permeability is exhibited.

In this paper, we study the effects of coupling in transmission lines loaded with pairs of SRRs magnetically coupled to the nearest neighboring pairs of resonators. The magnetic coupling between resonators of adjacent cells is thus accounted for in the model. Therefore, the resulting lumped element equivalent circuit model of the unit cell is a four-port circuit, in parallel to the four-port circuit that describes the unit cell of a CSRR-loaded line with interresonator coupling. The dispersion relation of SRR-loaded lines, inferred from Bloch mode analysis applied to the circuit model, was already derived in a previous publication by the authors. ${ }^{18}$ In this paper, further details on the derivation of such relation are given. Moreover, the modal solutions obtained theoretically are validated by obtaining the dispersion relation by means of a numerical eigenmode solver. Finally, we report the characterization of two fabricated structures with different level of coupling between SRRs, in order to experimentally confirm the effects of coupling on bandwidth enhancement. 


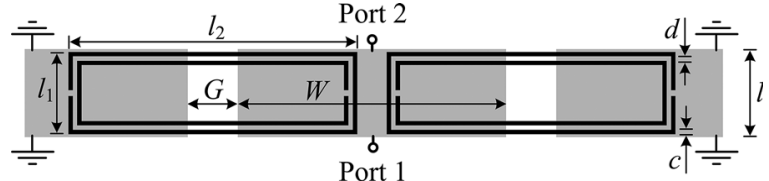

FIG. 1. Typical unit cell of a CPW transmission line loaded with a pair of SRRs designed to enhance coupling between resonators of neighboring cells. Dimensions are: $W=9.1 \mathrm{~mm}, G=1.7 \mathrm{~mm}, l=3 \mathrm{~mm}, c=d=0.15 \mathrm{~mm}$, $l_{1}=2.8 \mathrm{~mm}$, and $l_{2}=9.8 \mathrm{~mm}$. The considered substrate is Rogers $R O 3010$ with thickness $h=1.27 \mathrm{~mm}$ and dielectric constant $\varepsilon_{r}=11.2$. The Bloch wave propagates from port 1 to port 2.

\section{TOPOLOGY AND CIRCUIT MODEL OF THE SRR-LOADED LINES}

The topology of the considered SRR-loaded lines (unit cell) is depicted in Fig. 1. It consists of a coplanar waveguide (CPW) transmission line loaded with pairs of rectangular SRRs etched on the back side of the substrate. It is important to highlight that the symmetry plane of the SRRs (crossing the gaps) is orthogonally oriented to the line axis. This orientation is necessary to guarantee that the line is only capable of exciting the SRR fundamental resonance through the magnetic coupling. With different orientations, mixed coupling is required for an accurate description of the structure, as discussed and reported in Ref. 19. Obviously, mixed coupling between the line and resonators makes the analysis of SRRloaded lines with inter-resonator coupling much more cumbersome, and, for this reason, we have considered such SRR orientation in the present study. Thus, with this SRR orientation, the lumped-element equivalent circuit model of these structures, including the magnetic coupling between SRRs of neighboring cells, is depicted in Fig. 2(a) (the nearestneighbor interaction approximation is considered, and losses are neglected).
The validity of the model is restricted to those frequencies where the resonators are electrically small enough; this extends up to frequencies beyond the SRR fundamental resonance, the region of interest. In the model, $L$ and $C$ are the per-section line inductance and capacitance, respectively; the SRR is described by the capacitance $C_{s}$ and the inductance $L_{s} ; M$ is the mutual inductance between the line and the SRRs; finally, inter-resonator coupling is accounted for through the mutual inductance $M_{R}$. Note that the magnetic coupling between coplanar SRRs of adjacent cells is negative, and the proper modeling of the magnetic coupling sign is mandatory, i.e., the sign cannot be disregarded. Otherwise, the frequency response predicted by the circuit will not be able to describe correctly the behavior of the SRR-loaded lines. It is also important to highlight that when the SRRs of the same unit cell are close together, there can be positive magnetic coupling between them. However, this coupling is neglected here because its only effect is to decrease the resonance frequency.

In order to simplify the analysis of the circuit model, it is convenient to combine the parallel connection of the SRRs belonging to the same unit cell and to transform each pair of inductances coupled by the mutual inductance $M_{R}$ to its equivalent T-circuit. ${ }^{20}$ This leads us to the circuit of Fig. 2(b), from which it is possible to identify the four-port unit cell of Fig. 2(c). Qualitatively, this unit cell is composed of a conventional transmission line-type circuit magnetically coupled to a secondary propagating structure. This latter structure supports a kind of backward waves that have been studied in depth in Refs. 21-26 and have been called magnetoinductive waves (MIWs). MIWs propagate within a narrow frequency band in the vicinity of SRR resonance, and the associated bandwidth is dependent on how strongly the resonators are coupled to each other. The stronger is the a

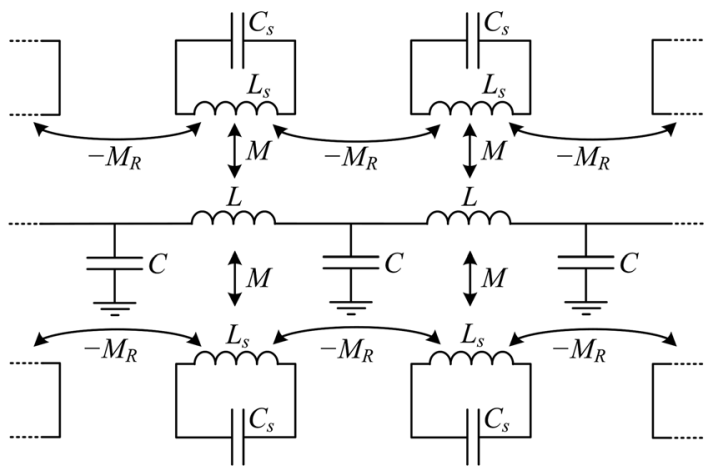

b

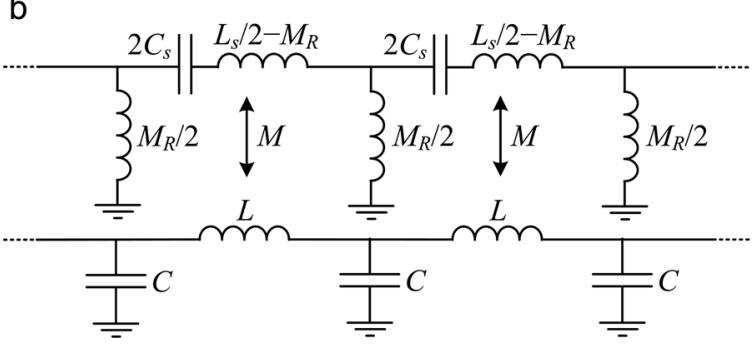

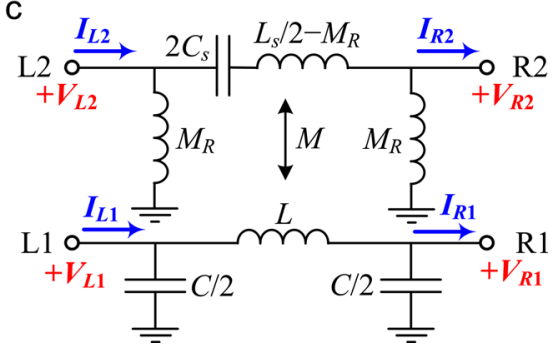

FIG. 2. Lumped-element equivalent circuit model of a periodic structure composed of an array of unit cells as the ones shown in Fig. 1, including magnetic coupling between resonators of adjacent cells; (a) model with mutual inductance between resonators, (b) transformed model, and (c) four-port unit cell model indicating the port voltages and currents relative to the calculation of the transfer $A B C D$ matrix. (a) and (b) are reprinted with permission from Naqui $e t$ al., International Conference on Electromagnetics Advanced Applications (ICEAA), Copyright 2013 by IEEE. ${ }^{18}$ 
interaction between the resonant elements, the wider is the passband of the MIWs. ${ }^{26}$ As multiconductor theory predicts that the resulting unit cell can propagate two modes, ${ }^{27}$ forward and backward waves are expected to coexist at some frequency band.

\section{BLOCH MODE ANALYSIS AND DISPERSION RELATION}

The dispersion characteristics of these SRR-loaded lines can be obtained from Bloch mode theory applied to the fourport network of Fig. 2(c). Let us denote $V_{L i}$ and $I_{L i}$ as the voltages and currents at the ports $(i=1,2)$ of the left hand-side of the unit cell, and $V_{R i}$ and $I_{R i}$ as the variables at the right handside ports. The variables at both sides of the network are linked through a generalized order- 4 transfer matrix, according to

$$
\left(\begin{array}{c}
V_{L} \\
I_{L}
\end{array}\right)=\left(\begin{array}{cc}
A & B \\
C & D
\end{array}\right)\left(\begin{array}{c}
V_{R} \\
I_{R}
\end{array}\right)
$$

where $\boldsymbol{V}_{\boldsymbol{L}}, \boldsymbol{I}_{\boldsymbol{L}}, \boldsymbol{V}_{\boldsymbol{R}}$, and $\boldsymbol{I}_{\boldsymbol{R}}$ are column vectors composed of the pair of port variables, and $\boldsymbol{A}, \boldsymbol{B}, \boldsymbol{C}$, and $\boldsymbol{D}$ are order-2 matrices.

The dispersion relation is obtained from the eigenmodes of the system (1)

$$
\operatorname{det}\left(\begin{array}{cc}
\boldsymbol{A}-e^{\gamma l} \cdot \boldsymbol{I} & \boldsymbol{B} \\
\boldsymbol{C} & \boldsymbol{D}-e^{\gamma l} \cdot \boldsymbol{I}
\end{array}\right)=0
$$

where $\boldsymbol{I}$ is the identity matrix, the propagation factor $\mathrm{e}^{\gamma l}$ is the eigenvalue, $\gamma=\alpha+\mathrm{j} \beta$ is the complex propagation constant, and $l$ is the unit cell length. For reciprocal, lossless, and symmetric networks, the eigenvalues can be simplified to the solutions of 28,29

$$
\operatorname{det}(\boldsymbol{A}-\cosh (\gamma l) \cdot \boldsymbol{I})=0
$$

which gives

$$
\cosh (\gamma l)=\frac{1}{2}\left(A_{11}+A_{22} \pm \sqrt{\left(A_{11}-A_{22}\right)^{2}+4 A_{12} A_{21}}\right),
$$

where the elements of the $\boldsymbol{A}$ matrix (inferred from the network of Fig. 2(c) as detailed in Appendix A) are

$$
\boldsymbol{A}=\boldsymbol{D}^{t}=\left(\begin{array}{cc}
1-\frac{L C}{2} \omega^{2} & \frac{M}{M_{R}} \\
-\frac{M C}{2} \omega^{2} & \frac{1}{2 M_{R}}\left(L_{s}-\frac{1}{C_{s} \omega^{2}}\right)
\end{array}\right)
$$

Since the network of Fig. 2(c) is lossless, the elements of $\boldsymbol{A}$ $\left(A_{i j}\right)$ are real numbers. Hence, if the expression under the square-root in (4) is positive, the propagation constant is either purely real $(\alpha \neq 0, \beta=0)$ or purely imaginary $(\alpha=0, \beta \neq 0)$, corresponding to evanescent or propagating modes, respectively. However, if that expression is negative, the two solutions are of the form $\gamma=\alpha \pm \mathrm{j} \beta$, corresponding to complex modes. The frequency band that supports complex modes is thus obtained by forcing the expression under the square-root in (4) to be negative. Since complex modes do not carry net power, the frequency band supporting such modes is a rejection band, despite of being of different nature than that associated to evanescent modes (where $\alpha \neq 0, \beta=0$ ).

Inspection of (4) and (5) reveals that a necessary condition for the presence of complex modes is that $M$ is different from zero (this is always the case, unless the substrate of the considered $\mathrm{CPW}$ is extremely thick). Notice that $M=0$ means that the host line and the SRR array are decoupled. Under this situation, the second term of the expression under the square-root in (4) is null, and hence, the square root is a real number, preventing the appearance of complex modes. Indeed, the two modal solutions for $M=0$ are

$$
\cosh (\gamma l)=A_{11}=1-\frac{L C}{2} \omega^{2}
$$

corresponding to the dispersion relation of a lossless transmission line described by the well-known LC ladder network, and

$$
\cosh (\gamma l)=A_{22}=\frac{1}{2 M_{R}}\left(L_{s}-\frac{1}{C_{s} \omega^{2}}\right),
$$

that is, the dispersion of an array of inductively (edge) coupled SRRs, where MIWs are supported in a narrow frequency band in the vicinity of SRR resonance. Note that MIWs can exist as long as the reactance of the series resonator (between the ports L2 and R2) is capacitive and, obviously, this is another condition for supporting complex modes.

The dispersion relation (4) in the limit $M_{R} \rightarrow 0$, corresponding to negligible inter-resonator coupling, is also interesting to obtain. Under these conditions, the following result arises (see Appendix B):

$$
\cosh (\gamma l)=1-\frac{L C}{2} \omega^{2}+\frac{M^{2} C \omega^{2}}{\left(L_{s}-\frac{1}{C_{s} \omega^{2}}\right)} .
$$

This is the dispersion relation of an SRR-loaded line without coupling between SRRs, which can be inferred from the biport model reported in Ref. 30 [and depicted in Fig. 3(b)] by applying Bloch mode analysis (see also Appendix B).

Although low-loss microwave substrates and very lowresistivity conductors are used, some losses are always present in real SRR-loaded lines. However, the dispersion relation given by (4) can still be considered as a reasonable approximation to the actual dispersion of the structure. Indeed, the analysis excluding losses suffices for the purposes of this

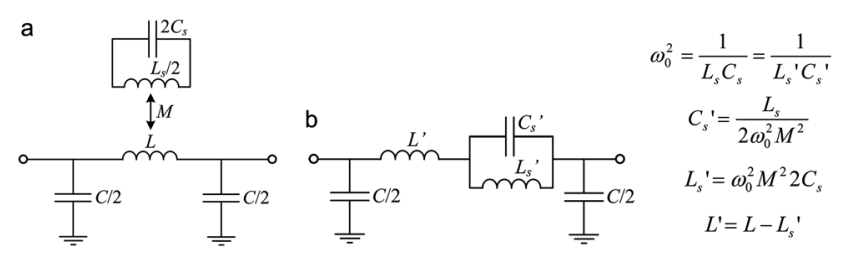

FIG. 3. Lumped element equivalent circuit model (unit cell) of the structure in Fig. 1 without inter-resonator coupling (a), and the corresponding transformed model (b). Reprinted with permission from Naqui et al., International Conference on Electromagnetics Advanced Applications (ICEAA), Copyright 2013 by IEEE. ${ }^{18}$ 

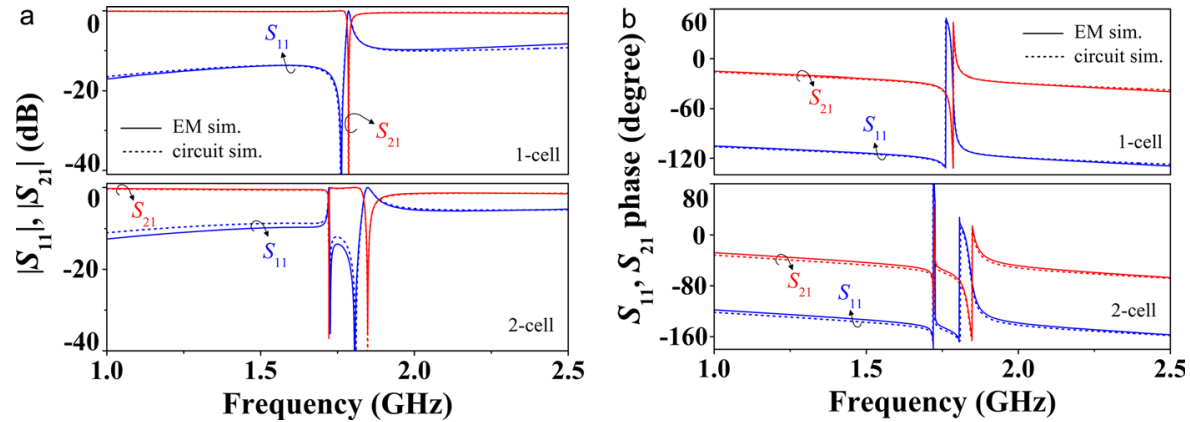

FIG. 4. Magnitude (a) and phase (b) of the lossless transmission $\left(S_{21}\right)$ and reflection $\left(S_{11}\right)$ coefficients for a unit cell and for two cascaded unit cells of the structure in Fig. 1. The extracted circuit parameters are: $L=1.01 \mathrm{nH}, C=1.40 \mathrm{pF}, L_{s}=17.66 \mathrm{nH}, C_{s}=0.45 \mathrm{pF}, M=0.72 \mathrm{nH}$, and $M_{R}=1.17 \mathrm{nH}$. (a) is reprinted with permission from Naqui et al., International Conference on Electromagnetics Advanced Applications (ICEAA), Copyright 2013 by IEEE. ${ }^{18}$

paper, i.e., the investigation of the effects of inter-resonator coupling on the dispersion characteristics and frequency response. In any case, the effects of losses may be easily evaluated by merely including resistors in the circuit model.

It is also worth mentioning that the dispersion characteristics of transmission lines loaded with SRRs (considering coupled and decoupled SRRs) and metallic rods was analyzed in Ref. 23. It was proven that interaction between MIWs propagating through an array of SRRs and incident electromagnetic waves (modeled by the equivalent circuit of a transmission line) may exist leading to the appearance of a stopband. Subsequently, an extended analysis was reported in Ref. 6 without the rods (i.e., by considering the same structure as in this work), and that stopband was found to be due to the presence of complex modes. In comparison to Refs. 6 and 23, in this paper, we obtain the dispersion relation from the multiport equivalent circuit providing the details of the calculation of the transfer matrix, and we consider a real device based on planar transmission lines and rings, instead of assuming a theoretical generic system. Moreover, we provide numerical results inferred from an eigenmode solver and experimental evidence of stopband bandwidth enhancement caused by inter-resonator coupling.

\section{A. Parameter extraction and equivalent circuit model validation}

We have extracted the circuit parameters of the structure of Fig. 1. To this end, the electromagnetic simulation of an isolated unit cell was performed (by the Agilent Momentum commercial software). The circuit model of a decoupled unit cell is depicted in Fig. 3(a). We have extracted the circuit elements of the transformed model of Fig. 3(b) following the procedure reported in Ref. 30. Then, $L_{s}$ has been estimated as the self-inductance of an isolated (without the CPW structure) single split ring with the same average radius and ring width as the considered SRRs (in a quasi-static approximation, the total current flowing on the pair of SRR rings is independent of the position on the $\mathrm{SRR}^{5}$ ). Thus, the equivalent inductance seen between the end terminals of the single split ring has been extracted from the electromagnetic simulation. By using the estimated $L_{s}(17.66 \mathrm{nH})$, the circuit elements of Fig. 3(a) have been obtained from the indicated transformation equations. Finally, we have inferred the mutual inductance $M_{R}$ of the circuits of Fig. 2 by curve fitting the circuit simulation to the electromagnetic simulation of a 2-cell structure. It is important to realize that since the SRRs of the input/output cells are not externally fed, the ports L2 (input cell) and R2 (output cell) have been left opened. Therefore, the transmission and reflection coefficients are referred to a two-port circuit (L1 and R1) rather than to the four-port circuit of the proposed model.

The extracted parameters are listed in the caption of Fig. 4. The comparison between the electromagnetic and circuit simulations of a unit cell and of two cascaded unit cells is depicted in Fig. 4, where good agreement is observed in the transmission and reflection coefficients. Concerning the modeling of higher order structures, Fig. 5(a) shows the frequency response for nine cascaded unit cells. As can be seen,
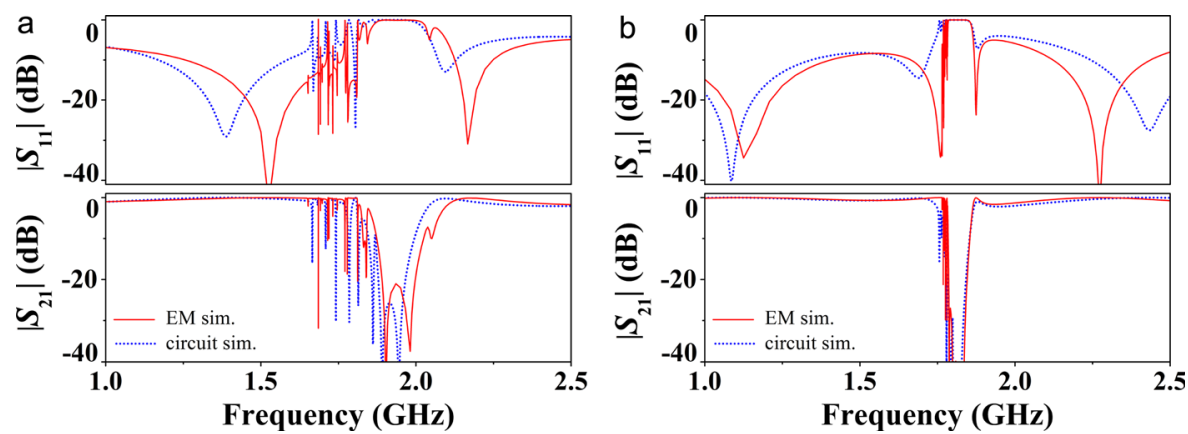

FIG. 5. Lossless electromagnetic and circuit simulations of the transmission and reflection coefficients (magnitude) for nine cascaded cells of the structure in Fig. 1 for (a) $l=3 \mathrm{~mm}$ and (b) $l=4.8 \mathrm{~mm}$ (the inter-resonator separation has been increased from $0.2 \mathrm{~mm}$ to $2 \mathrm{~mm}$ ). The circuit parameters are those indicated in the caption of Fig. 4, with the exception of $M_{R}=0.22 \mathrm{nH}$ in (b). Furthermore, in (b), a CPW section of $1.8 \mathrm{~mm}$ length has been cascaded between the port $\mathrm{R} 1$ and the port L1 of the contiguous cell in the circuit model. 

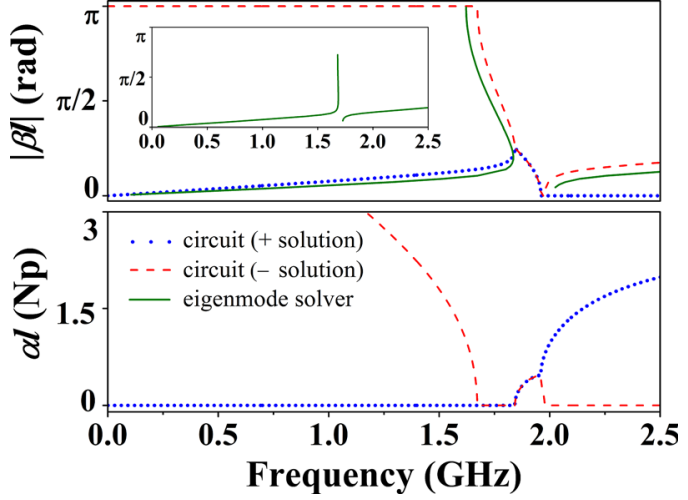

FIG. 6. Dispersion diagram for the structure of Fig. 1 inferred from an eigenmode solver and from its equivalent circuit model of Fig. 2(c). The circuit parameters are those indicated in the caption of Fig. 4. The dispersion diagram for the structure of Fig. 1 with $l=4.8 \mathrm{~mm}$ is depicted in the inset. The attenuation constant $\alpha$ is not provided by the eigenmode solver.

the central frequency of the stopband shifts upwards as the number of cells increases, being this effect produced by the negative inter-resonator coupling. It can also be observed that the stopband inferred from the electromagnetic simulation is even slightly more shifted than the one predicted by the circuit simulation. It has been found (by including additional couplings between non-adjacent cells) that this discrepancy is due to the assumption of first neighbor approximation. In this regard, as Fig. 5(b) confirms, an increase in the inter-resonator distance reduces the impact on the frequency response shift caused by such approximation. In any case, the first neighbor approximation suffices for the purpose of the present work.

It is also important to point out that inter-resonator coupling splits the SRR resonance frequencies, so that the number of transmission zeros equals the number of resonators. Moreover, the stronger the coupling, the stronger will be the the splitting. ${ }^{20}$ As a result, the stopband bandwidth broadens with inter-resonator coupling, although this enhancement is limited since it saturates with relatively few cells. For instance, the stopband bandwidth (computed at $-20 \mathrm{~dB}$ ) obtained from the circuit simulation for an order-9 structure [Fig. 5(a)] ranges from $1.879 \mathrm{GHz}$ to $1.966 \mathrm{GHz}$. This corresponds to a bandwidth similar to the maximum achievable bandwidth that will be given by the dispersion relation for an infinite structure in subsection III B.

\section{B. Dispersion relation validation}

Once the circuit parameters have been extracted, we can obtain the pair of modal propagation constants given by
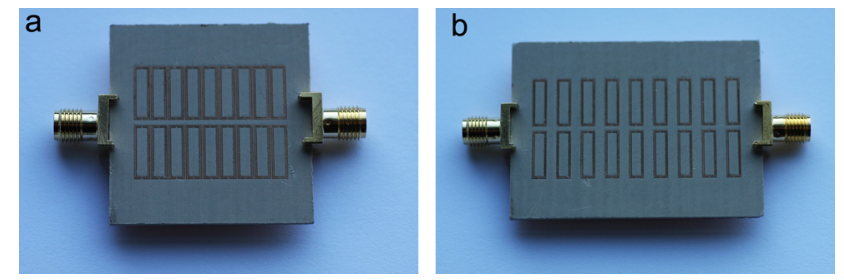

FIG. 7. Photograph of the bottom face of the fabricated order-9 structures composed of the unit cell in Fig. 1 with (a) $l=3 \mathrm{~mm}$ and (b) $l=4.8 \mathrm{~mm}$.

expression (4). The results are depicted in Fig. 6. As in Ref. 8 , in the first allowed band, there is a region with bi-valued propagation constant: one (forward) corresponding to transmission-line type propagation and the other (backward) related to magnetoinductive waves. Then, a region with a pair of conjugate complex propagation constants (complex modes) appears where forward and backward waves interfere with each other, followed by a region of evanescent waves. Finally, a forward wave transmission band emerges again. Hence, the enhancement of the stopband due to interresonator coupling is explained by the appearance of complex modes in the low frequency region of that stopband (the complex modes exist from $1.843 \mathrm{GHz}$ to $1.961 \mathrm{GHz}$, and the evanescent modes extends up to $1.977 \mathrm{GHz}$ ). However, the magnetic coupling between SRRs of adjacent cells is limited and so it is bandwidth broadening.

We have also obtained the dispersion relation of a periodic structure composed of a cascade of the unit cell in Fig. 1 by means of the full-wave eigenmode solver of CST Microwave Studio. The results, also depicted in Fig. 6, reveal that there is good agreement with the analytical dispersion curve predicted by the circuit model (the bi-valued region is perfectly predicted by the eigenmode solver). However, since there is no electromagnetic field pattern with net current transfer in the stopband, the tool is not able to provide the dispersion curves in that region.

For comparison purposes, we have also considered a structure with higher inter-resonator distance providing much weaker coupling. The dispersion diagram, depicted in the inset of Fig. 6, reveals that the stopband bandwidth is significantly narrower. Therefore, these dispersion diagrams indicate that most of the stopband in the structure of Fig. 1 is related to the presence of complex, rather than evanescent, modes. In other words, as long as inter-resonator coupling is significant, complex modes may be the dominant mechanism of signal rejection (in the vicinity of SRR fundamental resonance) of these SRR-loaded structures.
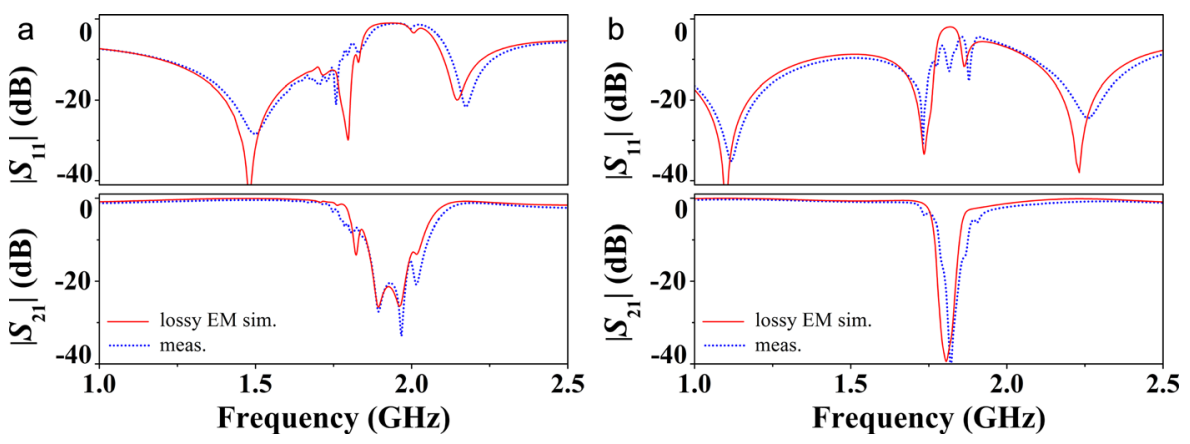

FIG. 8. Measurement and lossy electromagnetic simulation of the transmission and reflection coefficients (magnitude) of the structures in Fig. 7 ; (a) $l=3 \mathrm{~mm}$ and (b) $l=4.8 \mathrm{~mm}$. The loss tangent in Rogers $R O 3010$ is $\tan \delta=0.0023$. 


\section{EXPERIMENTAL RESULTS}

To experimentally validate the effects of inter-resonator coupling on bandwidth enhancement, two order-9 structures have been fabricated (Fig. 7): One of them with the unit cell of Fig. 1 ; the other by considering $l=4.8 \mathrm{~mm}$. The measured transmission and reflection coefficients are in good agreement with those given by the lossy electromagnetic simulation (see Fig. 8). The measured fractional stopband bandwidth (computed at $-20 \mathrm{~dB}$ ) is $5.2 \%$ and $2.4 \%$ for the structures of Figs. 7(a) and 7(b), respectively. The measured stopbands are also in accordance with those obtained from the dispersion relation. Hence, the dispersion relation inferred from the multi-terminal circuit model is a powerful tool to gain insight into the stopband and the effects of interresonator coupling in SRR-loaded lines.

\section{CONCLUSIONS}

It has been demonstrated that SRR-loaded lines with tightly coupled resonators exhibit forward (transmission line-type), backward (magnetoinductive-related), and complex modes. The structures have been analyzed using multiport Bloch mode theory applied to the lumped element equivalent circuit model, and the dispersion characteristics have been obtained. It has been found that complex modes are responsible for bandwidth enhancement of the stopband. These complex modes have been interpreted as the destructive interference between forward and backward waves. Since backward waves are supported by the chain of SRRs, inter-resonator coupling is absolutely mandatory for complex modes to emerge. Indeed, the behavior of SRR-loaded lines with strongly coupled resonators is very similar to that of CSRR-loaded lines. ${ }^{8}$ The main difference is the nature of the propagating waves in the frequency region that supports backward waves (bi-valued region with multimode forward and backward propagation). In CSRR-loaded lines, the backward waves are electroinductive-like waves, whereas in SRR-loaded lines, backward transmission is due to magnetoinductive waves. The theoretical results have been validated by means of a numerical eigenmode solver, able to provide the dispersion relation, and also by measuring the transmission and reflection characteristics of two fabricated SRRloaded lines (one with weak inter-SRR coupling, and the other one with significant coupling).

\section{ACKNOWLEDGMENTS}

This work has been supported by MICIIN-Spain (Contract No. TEC2010-17512, TEC2010-16948, CSD2008-00066, and TEC2011-13615-E), and by Generalitat de Catalunya (Project No. 2009SGR-421). Jordi Naqui has been awarded with a FPU Grant by MECD-Spain (Reference AP2010-0431).

\section{APPENDIX A: CALCULATION OF THE A MATRIX ELEMENTS}

The $\boldsymbol{A}$ matrix is an order-2 matrix that links the voltages of the pair of ports at both sides of the network of Fig. 2(c) under the condition $I_{R 1}=I_{R 2}=0$, that is,

$$
\left(\begin{array}{l}
V_{L 1} \\
V_{L 2}
\end{array}\right)=\left.\left(\begin{array}{ll}
A_{11} & A_{12} \\
A_{21} & A_{22}
\end{array}\right)\left(\begin{array}{c}
V_{R 1} \\
V_{R 2}
\end{array}\right)\right|_{I_{R 1}=I_{R 2}=0}
$$

From (A1), it follows that

$$
\begin{aligned}
& A_{11}=\left.\frac{V_{L 1}}{V_{R 1}}\right|_{V_{R 2}=0}, \\
& A_{22}=\left.\frac{V_{L 2}}{V_{R 2}}\right|_{V_{R 1}=0}, \\
& A_{12}=\left.\frac{V_{L 1}}{V_{R 2}}\right|_{V_{R 1}=0}, \\
& A_{21}=\left.\frac{V_{L 2}}{V_{R 1}}\right|_{V_{R 2}=0} .
\end{aligned}
$$

The derivation of $A_{11}$ is straightforward since conditions $I_{R 1}=I_{R 2}=0$ and $V_{R 2}=0$ means that there is not current flow across the inductance $L_{S} / 2-M_{R}$ in the circuit of Fig. 2(c). Therefore, we can write the voltage Kirchhoff's law between ports L1 and R1 (in the frequency domain) as

$$
V_{L 1}=j \omega L \cdot V_{R 1} \cdot j \omega \frac{C}{2}+V_{R 1} .
$$

From (A6), using (A2), the expression for $A_{11}$ given in (5) is obtained.

Similarly, $A_{22}$ is inferred from (A3) and

$$
V_{L 2}=\left(j \omega\left(\frac{L_{s}}{2}-M_{R}\right)+\frac{1}{j \omega 2 C_{s}}\right) \cdot V_{R 2} \cdot \frac{1}{j \omega M_{R}}+V_{R 2},
$$

which is simply the voltage Kirchhoff's law between ports L2 and R2 when $I_{R 1}=I_{R 2}=0$ and $V_{R 1}=0$.

To calculate $A_{12}$, the boundary conditions in the circuit of Fig. 2(c) are set to $I_{R 1}=I_{R 2}=0$ and $V_{R 1}=0$. Under these conditions, the voltage at port L1 can be expressed as

$$
V_{L 1}=j \omega M \cdot \frac{V_{R 2}}{j \omega M_{R}} .
$$

From (A8) and (A4), the result for $A_{12}$ shown in (5) is obtained.

Finally, to determine $A_{21}$, the circuit of Fig. 2(c) is subjected to $I_{R 1}=I_{R 2}=0$ and $V_{R 2}=0$. The voltage at port L2 is then found to be

$$
V_{L 2}=j \omega M \cdot V_{R 1} \cdot j \omega \frac{C}{2},
$$

and $A_{21}$ is deduced from (A9) and (A5).

\section{APPENDIX B: DISPERSION OF AN SRR-LOADED LINE IN THE LIMIT $M_{R} \rightarrow 0$}

By introducing the elements of (5) in (4), we obtain 


$$
2 \cosh (\gamma l)=1-\frac{L C}{2} \omega^{2}+\frac{1}{2 M_{R}}\left(L_{s}-\frac{1}{C_{s} \omega^{2}}\right) \pm \sqrt{\left[1-\frac{L C}{2} \omega^{2}-\frac{1}{2 M_{R}}\left(L_{s}-\frac{1}{C_{s} \omega^{2}}\right)\right]^{2}-\frac{2 M^{2} C \omega^{2}}{M_{R}}} .
$$

After some simple (but tedious) algebra, (B1) can be expressed as

$$
\begin{aligned}
2 \cosh (\gamma l)=1 & -\frac{L C}{2} \omega^{2}+\frac{1}{2 M_{R}}\left(L_{s}-\frac{1}{C_{s} \omega^{2}}\right) \pm \frac{1}{2 M_{R}}\left(L_{s}-\frac{1}{C_{s} \omega^{2}}\right) \\
& \times \sqrt{1+\frac{4 M_{R}^{2}\left(1-\frac{L C}{2} \omega^{2}\right)^{2}}{\left(L_{s}-\frac{1}{C_{s} \omega^{2}}\right)^{2}}-\frac{4 M_{R}\left(1-\frac{L C}{2} \omega^{2}\right)}{\left(L_{s}-\frac{1}{C_{s} \omega^{2}}\right)}-\frac{8 M^{2} M_{R} C \omega^{2}}{\left(L_{s}-\frac{1}{C_{s} \omega^{2}}\right)^{2}}} .
\end{aligned}
$$

Then, using the following Taylor series expansion up to the first order:

$$
\sqrt{1-x}=1-\frac{1}{2} x+\frac{1}{4} x^{2}-\cdots,
$$

expression (B2) can be approximated by

$$
\begin{aligned}
2 \cosh (\gamma l)= & 1-\frac{L C}{2} \omega^{2}+\frac{1}{2 M_{R}}\left(L_{s}-\frac{1}{C_{s} \omega^{2}}\right) \pm \frac{1}{2 M_{R}}\left(L_{s}-\frac{1}{C_{s} \omega^{2}}\right) \\
& \times\left\{1-\frac{2 M_{R}^{2}\left(1-\frac{L C}{2} \omega^{2}\right)^{2}}{\left(L_{s}-\frac{1}{C_{s} \omega^{2}}\right)^{2}}-\frac{2 M_{R}\left(1-\frac{L C}{2} \omega^{2}\right)}{\left(L_{s}-\frac{1}{C_{s} \omega^{2}}\right)}-\frac{4 M^{2} M_{R} C \omega^{2}}{\left(L_{s}-\frac{1}{C_{s} \omega^{2}}\right)^{2}}\right\},
\end{aligned}
$$

and by choosing the "_" sign (i.e., the solution with physical meaning) in the last term, we obtain expression (8).

To demonstrate that (8) is the dispersion relation corresponding to the two-port that models the unit cell of an SRRloaded line without inter-resonator coupling, we apply Bloch mode analysis to the model of Fig. 3(b), i.e.,

$$
\cosh (\gamma l)=A=1+\frac{Z_{s}}{Z_{p}},
$$

where $A$ is the first element of the transfer matrix of the twoport, and $Z_{s}$ and $Z_{p}$ are the impedances of the series and shunt branches of the network of Fig. 3(b). Calculation of (B5) for the circuit of Fig. 3(b) gives

$$
\cosh (\gamma l)=1-\frac{1}{2} L^{\prime} C \omega^{2}\left(1-\frac{1}{L^{\prime} C_{s}^{\prime} \omega^{2}\left(1-\frac{\omega_{0}^{2}}{\omega^{2}}\right)}\right),
$$

where $\omega_{o}=\left(L_{s} C_{s}\right)^{-1 / 2}$. Using the element transformations of Fig. 3, expression (B6) can be rewritten as

$$
\cosh (\gamma l)=1-\frac{1}{2} L C \omega^{2}+M^{2} C C_{s} \omega_{o}^{2} \omega^{2}+\frac{M^{2} C \omega_{o}^{2}}{L_{s}\left(1-\frac{\omega_{o}^{2}}{\omega^{2}}\right)},
$$

which, in turn, can be simplified to the dispersion relation shown in (8). Thus, it is clearly demonstrated that the general dispersion relation given in (4) for the four-port network of Fig. 2(c) is also able to account for the case of SRR-loaded lines with negligible inter-resonator coupling (i.e., $M_{R} \rightarrow 0$ ).

${ }^{1}$ J. B. Pendry, A. J. Holden, D. J. Robbins, and W. J. Stewart, IEEE Trans. Microwave Theor. Tech. 47, 2075 (1999).

${ }^{2}$ F. Martín, F. Falcone, J. Bonache, R. Marqués, and M. Sorolla, Appl. Phys. Lett. 83, 4652 (2003).

${ }^{3}$ F. Falcone, F. Martin, J. Bonache, R. Marqués, and M. Sorolla, Microwave Opt. Technol. Lett. 40, 3 (2004).

${ }^{4}$ D. R. Smith, W. J. Padilla, D. C. Vier, S. C. Nemat-Nasser, and S. Schultz, Phys. Rev. Lett. 84, 4184 (2000).

${ }^{5}$ R. Marqués, F. Martín, and M. Sorolla, Metamaterials with Negative Parameters: Theory, Design and Microwave Applications (John Wiley \& Sons, 2008).

${ }^{6}$ R. R. A. Syms and L. Solymar, Appl. Phys. Lett. 100, 124103 (2012).

${ }^{7}$ F. Martín, F. Falcone, J. Bonache, T. Lopetegi, R. Marqués, and M. Sorolla, IEEE Microwave Wireless Compon. Lett. 13, 511 (2003).

${ }^{8}$ J. Naqui, M. Durán-Sindreu, A. Fernández-Prieto, F. Mesa, F. Medina, and

F. Martín, IEEE Antennas Wireless Propag. Lett. 11, 1024 (2012).

${ }^{9}$ F. Falcone, T. Lopetegi, J. D. Baena, R. Marqués, F. Martín, and M. Sorolla, IEEE Microwave Wireless Compon. Lett. 14, 280 (2004).

${ }^{10}$ J. Naqui, A. Fernández-Prieto, M. Durán-Sindreu, F. Mesa, J. Martel, F. Medina, and F. Martín, IEEE Trans. Microwave Theor. Tech. 60, 3023 (2012).

${ }^{11}$ T. Tamir and A. A. Oliner, Proc. IEEE 110, 310 (1963).

${ }^{12}$ P. J. B. Clarricoats and K. R. Slinn, Electron. Lett. 1, 145 (1965).

${ }^{13}$ A. S. Omar and K. F. Schünemann, IEEE Trans. Microwave Theor. Tech. 34, 1508 (1986). 
${ }^{14}$ W. Huang and T. Itoh, IEEE Trans. Microwave Theor. Tech. 36, 163 (1988).

${ }^{15}$ M. J. Freire, F. Mesa, and M. Horno, IEEE Trans. Microwave Theor. Tech. 47, 1098 (1999).

${ }^{16} \mathrm{~F}$. Elek and G. V. Eleftheriades, IEEE Microwave Wireless Compon. Lett. 14, 434 (2004).

${ }^{17}$ R. Islam and G. V. Eleftheriades, IEEE Trans. Antennas Propag. 58, 1567 (2010).

${ }^{18}$ J. Naqui, M. Durán-Sindreu, F. Martín, A. Fernández-Prieto, F. Mesa, and F. Medina, International Conference on Electromagnetics Advanced Applications (ICEAA) (IEEE, 2013), p. 863.

${ }^{19}$ J. Naqui, M. Durán-Sindreu, and F. Martín, IEEE Antennas Wireless Propag. Lett. 12, 178 (2013).

${ }^{20}$ J.-S. Hong and M. J. Lancaster, Microstrip Filters for RF/Microwave Applications (John Wiley \& Sons, 2001).

${ }^{21}$ E. Shamonina, V. A. Kalinin, K. H. Ringhofer, and L. Solymar, Electron. Lett. 38, 371 (2002).
${ }^{22}$ E. Shamonina, V. A. Kalinin, K. H. Ringhofer, and L. Solymar, J. Appl. Phys. 92, 6252 (2002).

${ }^{23}$ R. R. A. Syms, E. Shamonina, V. Kalinin, and L. Solymar, J. Appl. Phys. 97, 064909 (2005).

${ }^{24}$ M. J. Freire, R. Marqués, F. Medina, M. A. G. Laso, and F. Martín, Appl. Phys. Lett. 85, 4439 (2004).

${ }^{25}$ L. Solymar and E. Shamonina, Waves in Metamaterials (Oxford University Press, 2009).

${ }^{26}$ R. R. A. Syms, E. Shamonina, and L. Solymar, Eur. Phys. J. B 46, 301 (2005).

${ }^{27}$ R. Mongia, I. Bahl, and P. Barthia, RF and Microwave Coupled Line Circuits (Artech House, 1999).

${ }^{28}$ J. Shekel, Proc. IRE 42, 840 (1954).

${ }^{29}$ R. Islam, M. Zedler, and G. V. Eleftheriades, IEEE Trans. Antennas Propag. 59, 1562 (2011).

${ }^{30}$ F. Aznar, M. Gil, J. Bonache, L. Jelinek, J. D. Baena, R. Marqués, and F. Martín, J. Appl. Phys. 104, 114501 (2008). 